\title{
Modulation of the c-Jun N-terminal kinase activity in the embryonic heart in response to anoxia-reoxygenation: involvement of the $\mathrm{Ca}^{2+}$ and mitoK $_{\mathrm{ATP}}$ channels
}

\author{
Alexandre Sarre $\cdot$ Stéphany Gardier · \\ Fabienne Maurer · Christophe Bonny • \\ Eric Raddatz
}

Received: 24 December 2007/ Accepted: 28 March 2008

(C) Springer Science+Business Media, LLC. 2008

\begin{abstract}
Whether the response of the fetal heart to ischemia-reperfusion is associated with activation of the c-Jun N-terminal kinase (JNK) pathway is not known. In contrast, involvement of the sarcolemmal L-type $\mathrm{Ca}^{2+}$ channel (LCC) and the mitochondrial $\mathrm{K}_{\mathrm{ATP}}\left(\right.$ mitoK $\left._{\mathrm{ATP}}\right)$ channel has been established. This work aimed at investigating the profile of JNK activity during anoxiareoxygenation and its modulation by $\mathrm{LCC}$ and mitoK $_{\mathrm{ATP}}$ channel. Hearts isolated from 4-day-old chick embryos were submitted to anoxia $(30 \mathrm{~min})$ and reoxygenation (60 min). Using the kinase assay method, the profile of JNK activity in the ventricle was determined every $10 \mathrm{~min}$ throughout anoxia-reoxygenation. Effects on JNK activity of the LCC blocker verapamil $(10 \mathrm{nM})$, the mitoK $\mathrm{ATP}_{\mathrm{AT}}$ channel opener diazoxide $(50 \mu \mathrm{M})$ and the blocker 5-hydroxydecanoate (5-HD, $500 \mu \mathrm{M})$, the mitochondrial $\mathrm{Ca}^{2+}$ uniporter (MCU) inhibitor Ru360 $(10 \mu \mathrm{M})$, and the antioxidant $\mathrm{N}$-(2-mercaptopropionyl) glycine (MPG, $1 \mathrm{mM}$ ) were determined. In untreated hearts, JNK activity was increased by $40 \%$ during anoxia and peaked fivefold relative to basal level after 30-40 min reoxygenation. This peak value was reduced by half by diazoxide and was
\end{abstract}

A. Sarre and S. Gardier contributed equally to this work.

A. Sarre $\cdot$ S. Gardier · E. Raddatz $(\bowtie)$

Department of Physiology, Faculty of Biology and Medicine, University of Lausanne, 7 rue du Bugnon, 1005 Lausanne,

Switzerland

e-mail: eric.raddatz@unil.ch

A. Sarre

e-mail: alexandre.sarre@medecine.unige.ch

F. Maurer · C. Bonny

Service of Medical Genetics, Centre Hospitalier Universitaire

Vaudois and University of Lausanne, Lausanne, Switzerland tripled by 5-HD. Furthermore, the 5-HD-mediated stimulation of JNK activity during reoxygenation was abolished by diazoxide, verapamil or Ru360. MPG had no effect on JNK activity, whatever the conditions. None of the tested pharmacological agents altered JNK activity under basal normoxic conditions. Thus, in the embryonic heart, JNK activity exhibits a characteristic pattern during anoxia and reoxygenation and the respective open-state of $\mathrm{LCC}, \mathrm{MCU}$ and mitoK $\mathrm{K}_{\mathrm{ATP}}$ channel can be a major determinant of JNK activity in a ROS-independent manner.

Keywords JNK $\cdot$ mitoK $_{\text {ATP }}$ channel $\cdot$ Calcium channel . Anoxia-reoxygenation · Embryo

\section{Introduction}

The signaling pathways involved in the response of the fetal heart to inadequate oxygenation, resulting from transient maternal hypoxemia, reduction in uterine or umbilical blood flow, remain to be explored. We have previously characterized in detail the electrical and contractile disturbances induced by anoxia and reoxygenation in the embryonic heart model $[1,2]$ and found that a moderate inhibition of the sarcolemmal L-type calcium channel (LCC) [3] or activation of the mitochondrial ATP-sensitive potassium ( mitoK $_{\mathrm{ATP}}$ ) channel can improve postanoxic recovery [4]. Opening of the mitoK $\mathrm{ATP}_{\mathrm{AT}}$ channel is also involved in ischemic preconditioning of isolated embryonic ventricular myocytes [5] and adult heart [6].

Several studies have underlined the key role played by the mitogen-activated protein kinases (MAPKs) pathways [7] in myocardial ischemia and reperfusion, particularly the stress-activated c-Jun N-terminal kinase (JNK) [8, 9]. In 
the ventricle of the anoxic-reoxygenated embryonic chick heart, activity of the extracellular signal-regulated kinase (ERK) is not significantly altered and the profile of the p38 MAPK phosphorylation is not affected by opening of the mitoK $_{\text {ATP }}$ channel [10]. However, information regarding the JNK signaling pathway in the hypoxic fetal heart is lacking and a better understanding is especially required in the context of recent advances in developmental cardiology [11], fetal cardiac surgery [12] and research dealing with intrauterine programming [13]. Furthermore, as $\mathrm{Ca}^{2+}$ is one of the second messengers capable of modulating JNK activity [14], the cytosolic and mitochondrial $\mathrm{Ca}^{2+}$ overload induced by anoxia-reoxygenation in embryonic cardiomyocytes [15] could interfere with the JNK signaling pathway.

The aim of this work was to establish the profile of JNK activity in the ventricle of the embryonic heart during anoxia and reoxygenation and to investigate the possible link between JNK activity and the state of activation of the $\mathrm{Ca}^{2+}$ and mitoK ${ }_{\mathrm{ATP}}$ channels. The results suggest that LCC and mitochondrial $\mathrm{Ca}^{2+}$ and $\mathrm{K}_{\mathrm{ATP}}$ channels are involved in the modulation of JNK activity in the embryonic ventricle submitted to anoxia-reoxygenation.

\section{Materials and methods}

\section{Reagents}

Dimethylsulfoxide (DMSO), mitoK $\mathrm{ATP}_{\mathrm{ATP}}$ channel opener diazoxide and blocker 5-hydroxydecanoate (5-HD), radical scavenger N-(2-mercaptopropionyl)glycine (MPG), were purchased from Sigma (Sigma-Aldrich, Buchs, Switzerland). L-Type $\mathrm{Ca}^{2+}$ channel inhibitor verapamil (Isoptin ${ }^{\circledR}$ ) was from Abbott and mitochondrial $\mathrm{Ca}^{2+}$ uniporter (MCU) inhibitor Ru360 was purchased from Calbiochem (JURO Supply, Lucerne, Switzerland).

$[\gamma-33 \mathrm{P}] \mathrm{ATP}$ was from Amersham Biosciences and inhibitors of proteases from Roche Biosciences.

Preparation and in vitro mounting of the heart

Fertilized eggs from Lohman Brown hens were incubated during $96 \mathrm{~h}$ at $38^{\circ} \mathrm{C}$ and $95 \%$ relative humidity to obtain stage $24 \mathrm{HH}$ embryo (according to Hamburger and Hamilton [16]). Spontaneously beating hearts were carefully excised and placed in the culture compartment of an airtight stainless steel chamber. The chamber was equipped with two windows for observation and maintained under controlled conditions on the thermostabilized stage $\left(37.5^{\circ} \mathrm{C}\right)$ of an inverted microscope (IMT2 Olympus, Tokyo, Japan) as previously detailed [4]. Briefly, the culture compartment $(300 \mu \mathrm{l})$ was separated from the gas compartment by a $15 \mu \mathrm{m}$ transparent and gas-permeable silicone membrane (RTV 141, Rhône-Poulenc, Lyon, France). Thus, $\mathrm{pO}_{2}$ at the tissue level could be strictly controlled and rapidly modified (within less than $5 \mathrm{~s}$ ) by flushing high-grade gas of selected composition through the gas compartment. At this developmental stage, the heart lacks vascularization and the myocardial oxygen requirement is met exclusively by diffusion.

The standard $\mathrm{HCO}_{3} / \mathrm{CO}_{2}$ buffered medium was composed of (in mmol/l): $99.25 \mathrm{NaCl} ; 0.3 \quad \mathrm{NaH}_{2} \mathrm{PO}_{4} ; 10$ $\mathrm{NaHCO}_{3} ; 4 \mathrm{KCl} ; 0.79 \mathrm{MgCl}_{2} ; 0.75 \mathrm{CaCl}_{2} ; 8 \mathrm{D}+$ glucose. This culture medium was equilibrated in the chamber with $2.31 \% \mathrm{CO}_{2}$ in air (normoxia and reoxygenation) or in $\mathrm{N} 2$ (anoxia) yielding a $\mathrm{pH}$ of 7.4. All reagents were diluted in this medium containing $0.5 \%$ DMSO (vehicle).

\section{Anoxia-reoxygenation protocol}

After 45 min of in vitro stabilization under normoxia at $37.5^{\circ} \mathrm{C}$ (stab), the hearts were submitted to strict anoxia during $30 \mathrm{~min}$ and then reoxygenated during $60 \mathrm{~min}$. The pharmacological agents were present throughout anoxiareoxygenation. The hearts were collected every $10 \mathrm{~min}$ and the ventricle was carefully dissected on ice and stored at $-80^{\circ} \mathrm{C}$ for subsequent determinations. As control, in a separate set of experiments, hearts were maintained under steady normoxia for 60 and $90 \mathrm{~min}$ after stabilization, corresponding to the time points of 30 and $60 \mathrm{~min}$ of postanoxic reoxygenation, respectively. Ventricles of these untreated hearts were also dissected and stored at $-80^{\circ} \mathrm{C}$.

\section{Kinase assay}

Ventricular JNK activity was determined using a published method [17] with minor modifications. Ventricles were homogenized in ice-cold lysis buffer (in mmol/l: 20 Trisacetate (pH 7), 270 sucrose, 1 EGTA, 1 EDTA, $50 \mathrm{NaF}, 10$ $\beta$-glycerophosphate, 10 dithiothreitol (DTT), 10 4-nitrophenyl phosphate disodium salt hexahydrate (PNPP), $1 \%$ Triton X-100 and inhibitors of proteases). Insoluble material was removed by a 5 min centrifugation at $10,000 \mathrm{~g}$ and protein contents were measured by the method of Lowry [18] with bovine serum albumin as standard.

Soluble ventricular protein extract $(30 \mu \mathrm{g})$ were incubated for $3 \mathrm{~h}$ at $4^{\circ} \mathrm{C}$ in the presence of $1 \mu \mathrm{g}$ GST-c-Jun $(1-219)$ bound to gluthatione-agarose beads as both JNK-specific ligand and substrate. The beads were washed three times in washing buffer (same as lysis buffer but with $0.1 \%$ Triton $\mathrm{X}-100$ ) and twice in kinase buffer (in mmol/l, 20 HEPES $\mathrm{pH}$ 7.5, $10 \mathrm{MgCl}_{2}, 20 \beta$-glycerophosphate, 10 DTT, 10 PNPP and inhibitors of proteases). Kinase reaction was carried out for $30 \mathrm{~min}$ at $30^{\circ} \mathrm{C}$ in $20 \mu \mathrm{l}$ of kinase buffer containing $5 \mu \mathrm{Ci}$ 
[ $\gamma$-33P]ATP. Reaction products were resolved by $12 \%$ SDS-polyacrylamide gel electrophoresis (SDS-PAGE), gels were dried, and phosphorylation signals were visualized by autoradiography, quantified by PhosphoImager (Quantityone 1.4.0, Biorad) and expressed as fold increase relative to the respective preanoxic value (stab).

Statistical analysis

All values are reported as mean \pm standard error of the mean (S.E.). The significance of any difference between the groups was assessed using Student $t$-test. The statistical significance was defined by a value of $P<0.05$.

\section{Results}

Profile of JNK activity during anoxia-reoxygenation

In control conditions (vehicle), JNK activity increased by $40 \%$ after $10-20 \mathrm{~min}$ anoxia $(P<0.05)$. During reoxygenation, JNK activity progressively increased, peaking after 30-40 min and further declined (Fig. 1). This culmination of JNK activity was specifically related to reoxygenation, since it was not attributable to the conditions and the duration (135 $\mathrm{min})$ of culture in the chamber (Fig. 2).

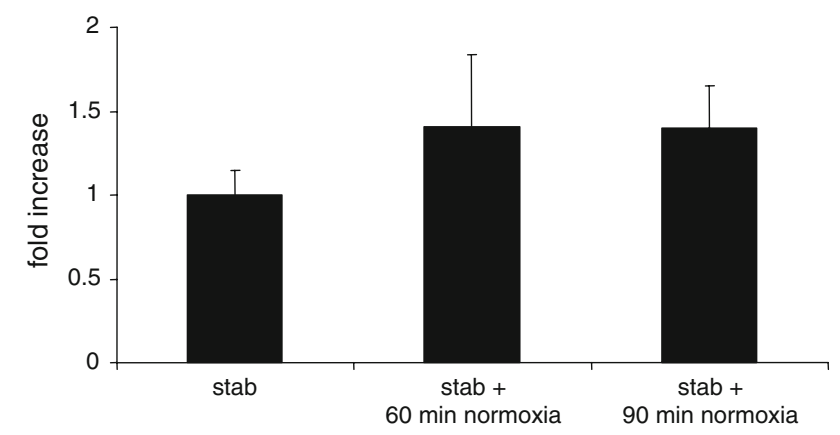

Fig. 2 Stability of JNK activity under normoxia. In vitro culture under steady normoxia did not affect ventricular JNK activity assessed by kinase assay. Fold increase: JNK activity is given relative to the mean of stab values. Stab: 45 min preanoxic stabilization; stab $+60 \mathrm{~min}$ and $\mathrm{stab}+90 \mathrm{~min}$ correspond to 30 and $60 \mathrm{~min}$ of reoxygenation in the anoxia-reoxygenation protocol, respectively (see Fig. 1). Mean \pm S.E.; $n=6-8$ determinations of JNK activity

Modulation of JNK activity by the opening state of the mitoK $\mathrm{ATP}_{\mathrm{AT}}$ channel

The mitoK $_{\mathrm{ATP}}$ channel opener diazoxide $(50 \mu \mathrm{mol} / \mathrm{l})$ decreased JNK activity after $30 \mathrm{~min}$ anoxia and throughout reoxygenation (Fig. 1). As the diazoxideinduced inhibition was the strongest at the peak of JNK activity $(P<0.02)$, we selected this time point to pharmacologically explore the possible mechanisms linking the open state of the mitoK $\mathrm{K}_{\mathrm{ATP}}$ channel with the JNK activity. Under preanoxic conditions, however, none of
Fig. 1 Profile of JNK activity in the embryonic ventricle during anoxia-reoxygenation. Activation of the mitoK $_{\mathrm{ATP}}$ channel by diazoxide decreased JNK activity. Upper panels show representative autoradiogram of JNK activity during anoxia and reoxygenation. Fold increase: JNK activity is given relative to the preanoxic stab value of vehicle. Stab: 45 min preanoxic stabilization; mean \pm S.E. of number of determinations indicated in columns; $* P<0.05$ vs. stab; ${ }^{\#} P<0.05$ vs. vehicle
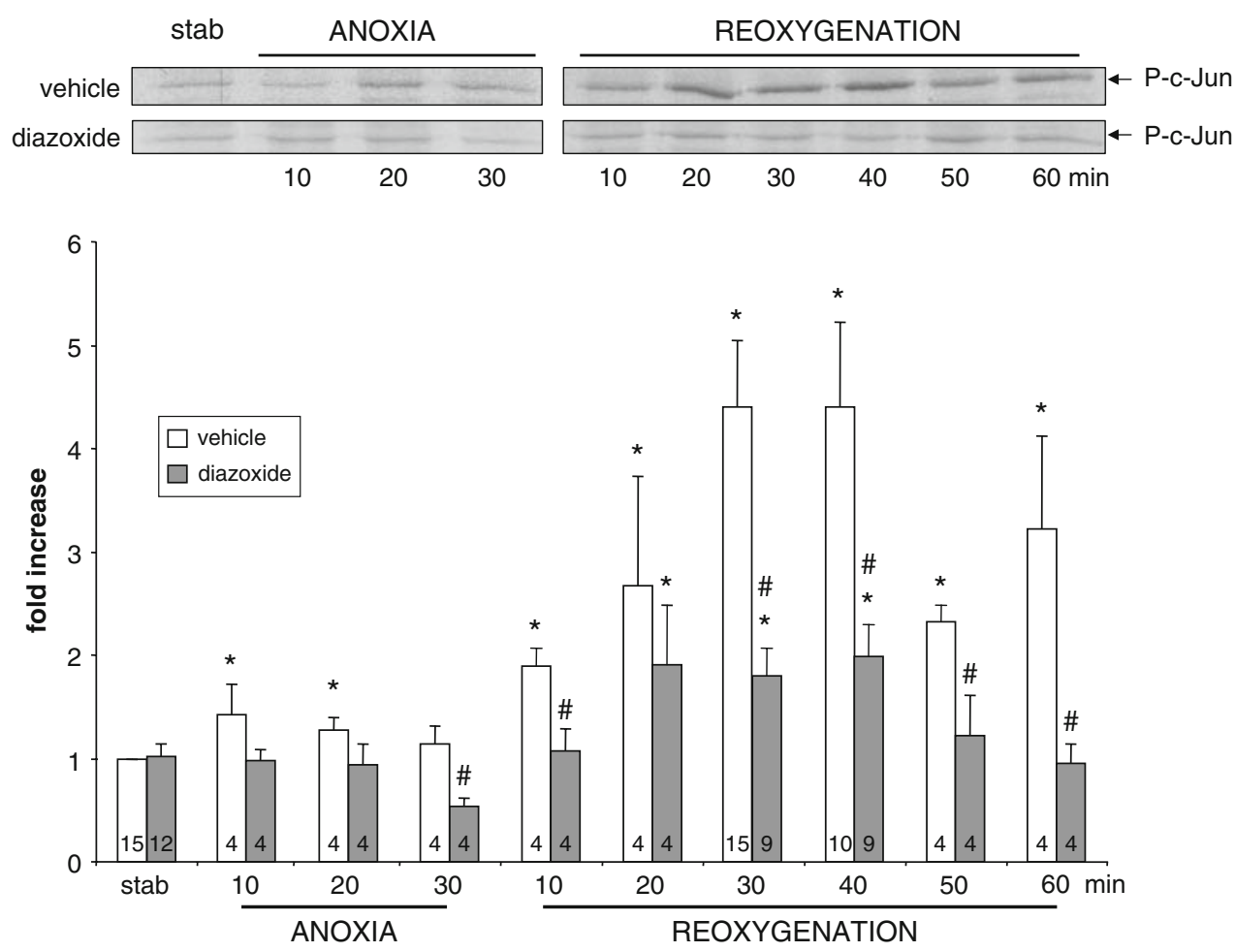

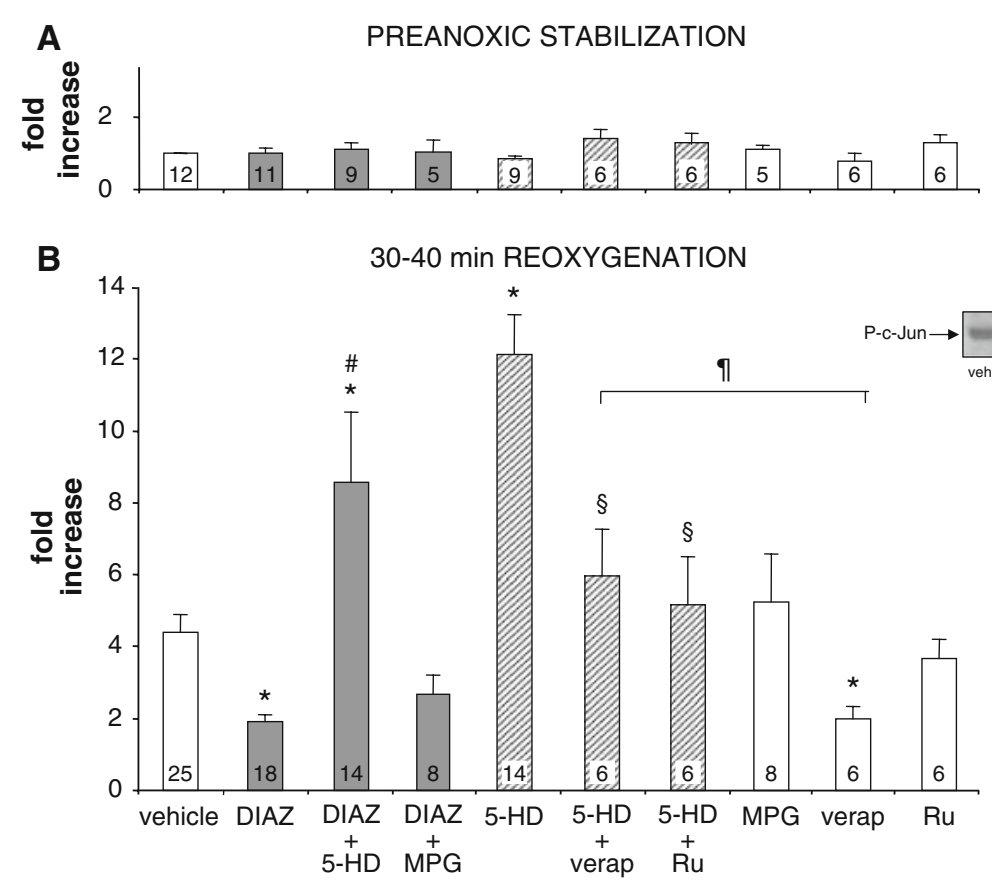

Fig. 3 Pharmacological modulation of JNK activity in the embryonic ventricle during preanoxic stabilization (a) and after 30-40 min reoxygenation (b). JNK activity was dependent on the open-state of the mitoK $\mathrm{K}_{\mathrm{ATP}}$ channel through $\mathrm{Ca}^{2+}$-dependent mechanisms during reoxygenation exclusively. In panel (a), JNK activity is given relative to the value of vehicle. In panel (b), JNK activity is given relative to the respective preanoxic stabilization value shown in panel (a). Insets show representative autoradiograms of JNK activity during reoxygenation in relation to $\mathrm{Ca}^{2+}$ handling. DIAZ: diazoxide; verap: verapamil; $\mathrm{Ru}$ : Ru360; Mean \pm S.E. of number of determinations indicated in columns; ${ }^{*} P<0.05$ vs. vehicle; ${ }^{\#} P<0.05$ vs. DIAZ; ${ }^{\S} P<0.05$ vs. 5 -HD; ${ }^{\circledR} P<0.055-\mathrm{HD}+$ verap vs. verap the reagents, alone or in combination, affected JNK activity relative to vehicle (Fig. 3a) or disturbed the regular contractile activity of the isolated hearts (not shown). After 30-40 min reoxygenation, inhibition of JNK activity by diazoxide was suppressed by the mitoK $_{\text {ATP }}$ channel blocker 5-HD $(500 \mu \mathrm{mol} / \mathrm{l})$ and, importantly, 5-HD alone tripled JNK activity with respect to vehicle $(P<0.01)$ (Fig. 3b). The radical scavenger MPG (1 mmol/l), known otherwise to abolish the diazoxide-induced ROS production and cardioprotection at reoxygenation [4], affected neither JNK activity nor the diazoxide-induced JNK inhibition.

Modulation of JNK activity by sarcolemmal (LCC) and mitochondrial (MCU) $\mathrm{Ca}^{2+}$ channels

Relative to vehicle, LCC inhibitor verapamil (10 nmol/l) decreased JNK activity by $55 \%$, whereas the mitochondrial $\mathrm{Ca}^{2+}$ uniporter inhibitor Ru360 (10 $\left.\mu \mathrm{mol} / \mathrm{l}\right)$ had no significant effect (Fig. 3b). However, the 5-HD-mediated JNK activity during reoxygenation was abolished by verapamil and also by Ru360. These observations indicate that $\mathrm{Ca}^{2+}$ entry is a prerequisite for JNK stimulation and that MCU is involved in JNK activation induced by the mitoK $\mathrm{ATP}_{\mathrm{AT}}$ channel blocker 5-HD.

\section{Discussion}

To the best of our knowledge, this is the first time that JNK activity is explored in the embryonic myocardium submitted to an anoxic episode. Our main findings indicate that JNK activity in the ventricle of the isolated embryonic heart (1) is increased by anoxia and reoxygenation, (2) is modulated by the open-state of the mitoK $\mathrm{ATP}_{\mathrm{AT}}$ channel, and (3) is dependent on $\mathrm{Ca}^{2+}$ flux through both LCC and MCU.

In the adult heart the effects of ischemia on JNK activation remain controversial whereas all studies show an enhanced JNK activity during reperfusion [8, 9, 19, 20]. Our data indicate that JNK pathway contributes to the short-term response of the heart to oxygen lack and reintroduction also during early embryogenesis, although the metabolic consequences of anoxia-reoxygenation differ to a certain extent from those of ischemia-reperfusion.

Although 5-HD has been shown to abolish ROS production induced by the mitoK $\mathrm{K}_{\mathrm{ATP}}$ channel opener diazoxide during reoxygenation [4], it markedly increased JNK activity (Fig. 3b). Furthermore, the membrane permeable antioxidant MPG which significantly reduces ROS production at reoxygenation [4] did not suppress JNK activation. Taken together, these observations clearly indicate that endogenous ROS are not prerequisite for JNK 
activation in the embryonic myocardium by contrast with neonatal cardiomyocytes [21] and adult heart [8]. Such a developmental discrepancy implies that JNK pathway can be modulated differently in prenatal and postnatal myocardium since, for example, the physiological oxygen level, the oxidative metabolism and the capacity to generate ROS are lower in embryonic tissues [22-24]. The facts that diazoxide improves recovery of atrio-ventricular conduction and E-C coupling during the first $20 \mathrm{~min}$ of reoxygenation [4], and reduces JNK activity from the end of anoxia onward (Fig. 1), suggest that this protection might be indirectly related to a reduction of JNK activity.

It has been shown that after 30 min reoxygenation, that is, when JNK activity reached its highest value (Fig. 1), $\mathrm{Ca}^{2+}$ uptake is maximally increased in embryonic ventricular cells [15] and contractility of the ventricle is transiently above its basal level [25], reflecting a rise of intracellular $\mathrm{Ca}^{2+}$. The present data support the concept that extracellular $\mathrm{Ca}^{2+}$ entry through LCC is a prerequisite for JNK activation in the reoxygenated embryonic myocardium (Fig. 3b) alike in neonatal [21] and adult cardiomyocytes [8].

Subcellular fractionation studies have shown that JNK can also be localized within or associated with mitochondrial structures [26, 27]. The facts that mitochondria are capable of taking up some of the cytosolic $\mathrm{Ca}^{2+}$ through the MCU in case of $\mathrm{Ca}^{2+}$ overload [28, 29] and that mitoK ${ }_{\mathrm{ATP}}$ channel opening can decrease the mitochondrial inner membrane potential $(\Psi \mathrm{m})$ and $\mathrm{Ca}^{2+}$ content in the matrix during hypoxia [30] could partly explain that MCU inhibition suppressed the 5-HD-induced JNK activation. Our results, indeed, show that blocking moderately $\mathrm{Ca}^{2+}$ entry through sarcolemmal LCC or blocking $\mathrm{Ca}^{2+}$ flux into mitochondrion through $\mathrm{MCU}$ reduces JNK activity during reoxygenation, especially when mitoK $_{\mathrm{ATP}}$ channel are closed by 5-HD.

Although $\Psi \mathrm{m}$ and mitochondrial $\mathrm{Ca}^{2+}$ concentration have not been measured because of the thickness of the tissue and interferences due to contractions, our findings support the hypothesis that during reoxygenation $\mathrm{Ca}^{2+}$ entry through LCC and/or influx through MCU can activate cytosolic JNK and/or JNK associated with mitochondria (see model proposed in Fig. 4).

When mitoK $_{\text {ATP }}$ channels are opened by diazoxide, $\Psi \mathrm{m}$ is known to drop [30, 31], thereby limiting mitochondrial $\mathrm{Ca}^{2+}$ entry through MCU and consequently $\mathrm{Ca}^{2+}$ overload, reducing JNK activation. Conversely, when mitoK $_{\text {ATP }}$ channels are blocked by 5-HD, $\Psi \mathrm{m}$ should be maintained and act as a driving force for $\mathrm{Ca}^{2+}$ transport through $\mathrm{MCU}$, which also could contribute to activate JNK.

Thus, in the embryonic heart, JNK activity exhibits a characteristic pattern during anoxia and reoxygenation and the respective open-state of $\mathrm{LCC}, \mathrm{MCU}$ and mitoK $_{\mathrm{ATP}}$

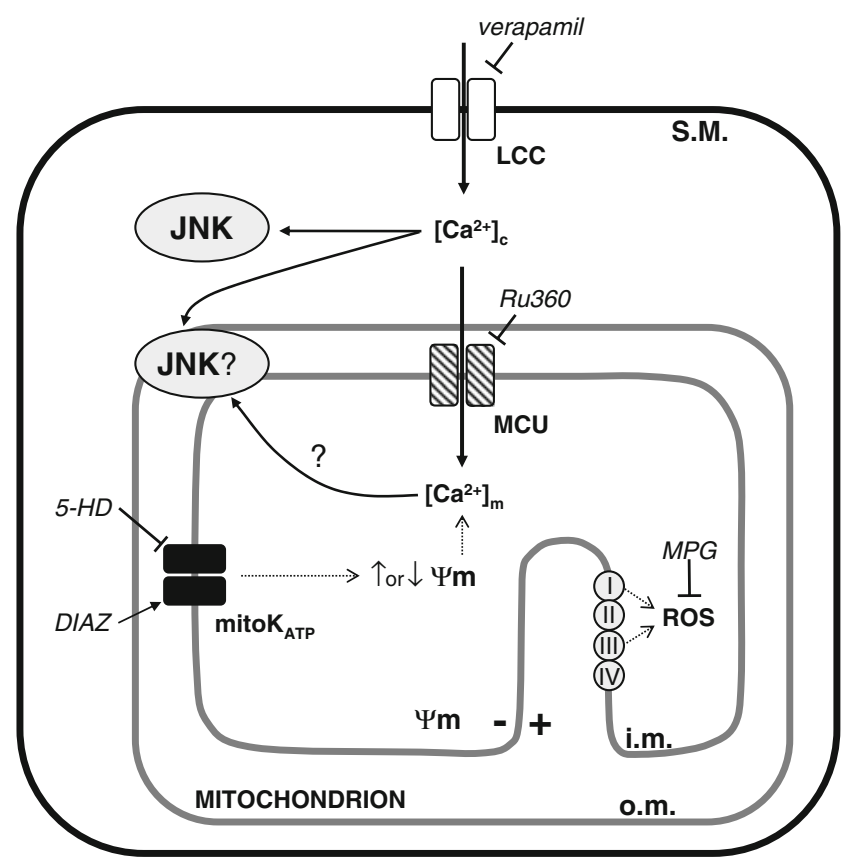

Fig. 4 Schematic representation based on the present findings and illustrating the possible modulation of JNK activity by open-state of L-type $\mathrm{Ca}^{2+}$ channel (LCC), mitochondrial $\mathrm{Ca}^{2+}$ uniporter (MCU) and mitoK $_{\text {ATP }}$ channel during reoxygenation of the embryonic ventricle. Inactivation of LCC (verapamil) decreased the reoxygenation-induced peak of JNK activity. Inhibition of MCU (Ru360) or LCC suppressed the 5-HD-induced JNK activity (see Fig. 3). Opening (DIAZ) and blockade (5-HD) of the mitoK $_{\mathrm{ATP}}$ channel could respectively decrease and increase $\Psi \mathrm{m},\left[\mathrm{Ca}^{2+}\right] \mathrm{m}$ and JNK activity. Intracellular antioxidant (MPG) had no effect on JNK activity. S.M.: sarcolemmal membrane; i.m. and o.m.: inner and outer mitochondrial membrane; $\Psi \mathrm{m}$ : i.m. potential; $\left[\mathrm{Ca}^{2+}\right] \mathrm{c}$ and $\left[\mathrm{Ca}^{2+}\right] \mathrm{m}$ : cytosolic and mitochondrial $\mathrm{Ca}^{2+}$ concentrations; MCU: mitochondrial $\mathrm{Ca}^{2+}$ uniporter; I-IV: respiratory chain complexes; ROS: reactive oxygen species

channels can be a major determinant of JNK activity in a ROS-independent manner. This work provides a first step in understanding the regulation of the JNK signaling pathway in the fetal heart transiently exposed to hypoxia. In particular, the cellular targets as well as the long-term functional consequences of an acute activation of this pathway during cardiogenesis deserve further investigations.

Acknowledgements We thank Anne-Catherine Thomas for her skilful technical assistance. This work was supported by the Swiss National Science Foundation n³100A0-105901.

\section{References}

1. Sarre A, Maury P, Kucera P et al (2006) Arrhythmogenesis in the developing heart during anoxia-reoxygenation and hypothermiarewarming: an in vitro model. $\mathrm{J}$ Cardiovasc Electrophysiol 17:1350-1359

2. Sedmera D, Kucera P, Raddatz E (2002) Developmental changes in cardiac recovery from anoxia-reoxygenation. Am J Physiol Regul Integr Comp Physiol 283:R379-R388 
3. Tenthorey D, de Ribaupierre Y, Kucera P et al (1998) Effects of verapamil and ryanodine on activity of the embryonic chick heart during anoxia and reoxygenation. J Cardiovasc Pharmacol 31:195-202

4. Sarre A, Lange N, Kucera P et al (2005) mitoKATP channel activation in the postanoxic developing heart protects E-C coupling via NO-, ROS-, and PKC-dependent pathways. Am J Physiol Heart Circ Physiol 288:H1611-H1619

5. Lebuffe G, Schumacker PT, Shao ZH et al (2003) ROS and NO trigger early preconditioning: relationship to mitochondrial KATP channel. Am J Physiol Heart Circ Physiol 284:H299H308

6. Garlid KD, Paucek P, Yarov-Yarovoy V et al (1997) Cardioprotective effect of diazoxide and its interaction with mitochondrial ATP-sensitive $\mathrm{K}+$ channels. Possible mechanism of cardioprotection. Circ Res 81:1072-1082

7. Schulz R, Cohen MV, Behrends M et al (2001) Signal transduction of ischemic preconditioning. Cardiovasc Res 52:181-198

8. Knight RJ, Buxton DB (1996) Stimulation of c-Jun kinase and mitogen-activated protein kinase by ischemia and reperfusion in the perfused rat heart. Biochem Biophys Res Commun 218:83-88

9. Omura T, Yoshiyama M, Shimada T et al (1999) Activation of mitogen-activated protein kinases in in vivo ischemia/reperfused myocardium in rats. J Mol Cell Cardiol 31:1269-1279

10. Gardier S, Sarre A, Thomas AC et al (2006) Activation of the mitoKATP channel differently modulates ERK, JNK and p38 MAPK in the ventricle of the anoxic-reoxygenated developing heart. Arch Mal Coeur Vaisseaux 99:376 (abstract)

11. Strasburger JF (2005) Prenatal diagnosis of fetal arrhythmias. Clin Perinatol 32:891-912

12. Kumar S, O'Brien A (2004) Recent developments in fetal medicine. BMJ 328:1002-1006

13. Fowden AL, Giussani DA, Forhead AJ (2006) Intrauterine programming of physiological systems: causes and consequences. Physiology (Bethesda) 21:29-37

14. Schaub MC, Hefti MA, Zaugg M (2006) Integration of calcium with the signaling network in cardiac myocytes. J Mol Cell Cardiol 41:183-214

15. Murphy JG, Smith TW, Marsh JD (1988) Mechanisms of reoxygenation-induced calcium overload in cultured chick embryo heart cells. Am J Physiol 254:H1133-H1141

16. Hamburger V, Hamilton H (1951) A series of normal stages in the development of the chick embryo. J Morphol 88:49-92

17. Larsen CM, Wadt KA, Juhl LF et al (1998) Interleukin-1betainduced rat pancreatic islet nitric oxide synthesis requires both the p38 and extracellular signal-regulated kinase 1/2 mitogenactivated protein kinases. J Biol Chem 273:15294-15300
18. Lowry O, Rosebrough N, Farr A et al (1951) Protein measurement with the Folin phenol reagent. J Biol Chem 193:265-275

19. Hreniuk D, Garay M, Gaarde W et al (2001) Inhibition of c-Jun $\mathrm{N}$-terminal kinase 1 , but not c-Jun N-terminal kinase 2, suppresses apoptosis induced by ischemia/reoxygenation in rat cardiac myocytes. Mol Pharmacol 59:867-874

20. Ren J, Zhang S, Kovacs A et al (2005) Role of p38alpha MAPK in cardiac apoptosis and remodeling after myocardial infarction. J Mol Cell Cardiol 38:617-623

21. Dougherty CJ, Kubasiak LA, Frazier DP et al (2004) Mitochondrial signals initiate the activation of c-Jun N-terminal kinase (JNK) by hypoxia-reoxygenation. Faseb J 18:1060-1070

22. Allen RG (1991) Oxygen-reactive species and antioxidant responses during development: the metabolic paradox of cellular differentiation. Proc Soc Exp Biol Med 196:117-129

23. Lebovitz RM, Zhang H, Vogel H et al (1996) Neurodegeneration, myocardial injury, and perinatal death in mitochondrial superoxide dismutase-deficient mice. Proc Natl Acad Sci USA 93:9782-9787

24. Romano R, Rochat AC, Kucera P et al (2001) Oxidative and glycogenolytic capacities within the developing chick heart. Pediatr Res 49:363-372

25. Rosa A, Maury JP, Terrand J et al (2003) Ectopic pacing at physiological rate improves postanoxic recovery of the developing heart. Am J Physiol Heart Circ Physiol 284:H2384-H2392

26. Horbinski C, Chu CT (2005) Kinase signaling cascades in the mitochondrion: a matter of life or death. Free Radic Biol Med 38:2-11

27. Wiltshire C, Gillespie DA, May GH (2004) Sab (SH3BP5), a novel mitochondria-localized JNK-interacting protein. Biochem Soc Trans 32:1075-1077

28. O'Rourke B, Cortassa S, Aon MA (2005) Mitochondrial ion channels: gatekeepers of life and death. Physiology (Bethesda) 20:303-315

29. Anderson CD, Pierce J, Nicoud I et al (2005) Modulation of mitochondrial calcium management attenuates hepatic warm ischemia-reperfusion injury. Liver Transpl 11:663-668

30. Kim MY, Kim MJ, Yoon IS et al (2006) Diazoxide acts more as a PKC-epsilon activator, and indirectly activates the mitochondrial K(ATP) channel conferring cardioprotection against hypoxic injury. Br J Pharmacol 149:1059-1070

31. Comelli M, Metelli G, Mavelli I (2007) Downmodulation of mitochondrial F0F1 ATP synthase by diazoxide in cardiac myoblasts: a dual effect of the drug. Am J Physiol Heart Circ Physiol 292:H820-H829 